A. Weisbecker, M. Burmester \& A. Schmidt (Hrsg.): Mensch und Computer 2015 Workshopband, Stuttgart: Oldenbourg Wissenschaftsverlag, 2015, S. 283-290.

\title{
Measuring Physical Pressure in Smart Phone Interaction for People with Impairments
}

\author{
Mirjam Augstein ${ }^{1}$, Daniel Kern², Thomas Neumayr ${ }^{2}$, Werner Kurschl ${ }^{3}$, Josef \\ Altmann ${ }^{1}$ \\ Communication and Knowledge Media, University of Applied Sciences Upper Austria ${ }^{1}$ \\ Research \& Development, University of Applied Sciences Upper Austria ${ }^{2}$ \\ Human-Centered Computing, University of Applied Sciences Upper Austria ${ }^{3}$
}

\begin{abstract}
Personalization has become an important aspect in human-computer interaction. Not only differ users drastically regarding their interests, knowledge and individual prerequisites, they also have varying interaction capabilities. The latter is especially significant for users with different kinds of cognitive and/or motor impairments. Thus, the IAAA project investigates novel interaction methods as well as methods to model users' interaction capabilities in order to personalize the overall interaction process later. This paper focuses on physical pressure as an additional method to interact with common smart phones which could also be used to interact with other interactive systems. It compares four different ways of measuring physical pressure for the specific domain and presents the results of an initial user study that has been conducted with the target group in order to i) evaluate how well users can handle the respective interaction method and ii) how reliably the different approaches work.
\end{abstract}

\section{Introduction}

Personalization has become a key aspect in human-computer interaction. Software and its operation should be adapted to the needs and capabilities of the individual user, especially if the target group is highly diverse. The IAAA project ${ }^{1}$ focuses on the automated personalization of interaction and involves research endeavors along two dimensions: it identifies and evaluates novel interaction modalities bearing potential to be useful for the

1 Interaction Analysis for Automated Adaptation. 
target group, and investigates user modeling and adaptation strategies to automate the personalization process. This paper focuses on the analysis of physical pressure applied to common smart phones as interaction method. An initial observation-based study with people with different kinds of impairments was conducted in autumn 2014. It revealed that many of the participants were only able to cover a small area of reach when using touch screens (also if the screen itself was small). Yet, most could control their movement along the vertical axis well which led to the hypothesis that physical pressure could be an appropriate way of interacting. This paper compares four technologically different ways of measuring physical pressure on common smart phones. The findings are decisive for the selection of a technology for the target group. We discuss related work in Section 2; Section 3 presents a technological comparison of the four approaches to measure pressure. Section 4 describes our user tests with people with different kinds of impairments; Section 5 provides a summary, conclusions and an outlook on subsequent work.

\section{Related Work}

Most of the work discussed here tries to overcome the limitation of the typical capacitive screen, which cannot be used for pressure sensing per se. We pick up on three approaches to compare their applicability in our specific target domain. In general, physical pressure can be captured in several different ways. One approach described by (Hwang, Hwang, Bianchi, Ahn, \& Wohn, 2013) uses a magnetically driven pen interface, where a pen is augmented with a magnet, where either the magnet is put on a fixed position, or the magnet rests on a spring embedded in the centre of the pen. The magnet's position related to the built-in magnetometer in the smart phone is used to $i$ ) identify a pen, ii) identify gestures drawn nearby, or iii) sense the pressure. An evaluation regarding the identification of pressure levels showed that the distance of the magnet must be considered accordingly, since the magnetic field decreases with the distance. A different approach using the magnetic field was developed by (Liang, Cheng, Su, Weng, Chen, \& Yang, 2012), who attached on the back of a device a sensor board with 192 hall-effect sensors. When the device's cover is made of any non-ferromagnetic material and is thinner than two cm, a pen can be used as an input device.

Another approach to sense pressure is described by (Hwang, Bianchi, \& Wohn, 2013), where the smart phone is brought to vibration with the built in vibration motor. When the device gets in contact with a damping surface (e.g., a user's hand), vibration absorption is measured with the built-in accelerometer. An evaluation showed that up to six pressure levels can be used, two of which lead to an accuracy of $99 \%$ for touch and grip. With increasing levels decreases the accuracy. A similar approach called GripSense was proposed by (Goel, Wobbrock, \& Patel, 2012) that distinguishes three pressure levels with an accuracy of $95.1 \%$. A usability test revealed that pressure input can be useful in numerous scenarios.

Another different approach to overcome the limitations of a capacitive touch screen proposed by (Hwang \& Wohn, 2012) is called PseudoButton. It tries to use an acoustic-based pressure-sensitive button, where a built-in speaker emits a sound, which cannot be heard by most humans (e.g., above $16.7 \mathrm{kHz}$ ). When a finger covers the pinhole of the microphone, it blocks and changes partially the sound according to the pressure applied by the finger. With a final signal analysis it is possible to identify up to five different pressure levels. The 
microphone as non-verbal sensor has also attracted researchers to use it for sound-based interaction (e.g., (Misra, Essl, \& Rohs, 2008) use it in a musical setting. However, microphones can also be used to derive positions as described by (Do \& Silverman, 2008).

As our research does not primarily focus on pressure detection methods but at making standard devices (like smart phones) better accessible for the target group, we did not consider additional hardware options (e.g., based on Force Resisting Sensors) as proposed by (Wilson, Stewart, \& Brewster, 2010) for our endeavors.

\section{Technologies and Smart Phone App}

We implemented four prototypes based on different technologies to measure physical pressure on common smart phones. The prototypes consist of test cases included in an Android app and were used to execute an initial evaluation with the target group (see Section 4). This section focuses on the technological characteristics. All four tests internally distinguish between twelve levels of pressure and display the currently applied pressure as a bar chart so that the user receives immediate visual feedback.

The vibration test measures pressure by analyzing vibration absorption as described by (Hwang, Bianchi, \& Wohn, 2013). Thus, our vibration test first activates the smart phone's vibration motor at the maximum level. The physical pressure applied by the user is then measured by evaluating the phone's built-in acceleration sensor's data. The acceleration sensor provides for each time stamp values for the three coordinates $(\mathrm{x}, \mathrm{y}, \mathrm{z})$, which are normalized and smoothed by our app before they are further processed. Next, the (Euclidean) distance between the coordinates at a certain time stamp and its predecessor are computed before the distances are added up (the smaller this sum, the higher the physical pressure; the maximum value defines the minimum pressure (mostly, no pressure).

The magnetic field test examines pressure by measuring changes in the magnetic field around the smart phone as described by (Hwang, Hwang, Bianchi, Ahn, \& Wohn, 2013). It analyzes the values for all three dimensions provided by the phone's built-in magnetic field sensor in $\mu \mathrm{T}$. These values are then used to compute the absolute magnetic field strength (which is next normalized and divided into different levels). Physical pressure in this case is not (as in the vibration test) applied to the phone itself but to an additional prototypic stylus we built. The stylus basically contains a magnet placed on a spring; it works like a common pen so that the user can press the top of it (hereby, the magnet is moved closer to the pen tip and its distance to the device is decreased). The magnetic field test faces additional challenges. In order to gain reliable results, the magnet's initial position must be predefined to calibrate the app accordingly. Thus, the screen indicates via displaying a red circle the position where the pen tip should be placed. As the magnetic field sensor might be installed at different positions for different devices, the optimum position can differ. This test is thus not so easily transferrable. Further, the magnetic field around a smart phone is prone to be influenced by external sources of disturbance (e.g., additional devices). Thus, in order to gain reliable results, the magnetic field must be analyzed before every test.

The microphone test evaluates physical pressure based on sound analysis as suggested by (Hwang \& Wohn, 2012). Our app outputs a sound of a specific frequency via the phone's 
built-in speakers and analyzes it using its microphone. Physical pressure is applied by covering the microphone (stronger pressure leads to better covering the microphone, thus the amplitude of the specific frequency is lowered). Several different frequencies were tested. It should be high enough not to be heard by at least a large number of people but still be easily recognizable by the device. The latter was particular challenging because different devices are able to recognize different frequencies. For instance, we tested a tablet ${ }^{2}$ which could only recognize frequencies of up to $10.000 \mathrm{~Hz}$ whereas a tested smart phone ${ }^{3}$ could recognize frequencies of up to $14.500 \mathrm{~Hz}$. For our app we used in the user tests, we set the frequency to 14.000 Hz. The app outputs the sound by repeatedly playing a sound file which contains a constant sound. The analysis of the output sound is done using Fourier transformation with a sampling rate of $44.100 \mathrm{~Hz}$ (in our implementation, the JTransforms library ${ }^{4}$ was used). The built-in touch test measures physical pressure by analyzing the user's touch input, based on information that is available via the standard Android SDK's methods getPressure and getSize. Depending on the device and display type, the touch point's size can be retrieved. The approach relies on the assumption that with increasing physical pressure, the size of the touch point increases too. A problem related to this approach is that the values for the touch point's size are strongly dependent on the device type. Devices have different built-in displays that support this approach to different degrees. In our user tests, a Samsung Galaxy S4 mini was used and the results were reasonable. However, for some devices there are limitations regarding processing data from touchscreen to low level driver to the higher level touch event. For these devices, the getPressure function returns a binary instead of a floating point value between 0 and 1 , which is not sufficient to measure pressure intensities.

\section{User Tests and Findings}

To evaluate the applicability of the chosen methods for our target group, initial user tests have been conducted in January 2015, using a Samsung Galaxy S4 mini ${ }^{5}$.

\subsection{Setting and Participants}

The first user test took place in a facility ${ }^{6}$ for people with impairments in Gallneukirchen, Upper Austria in a department where a group of people works with (new) media, e.g., publication of the facility's news magazine or taking and processing pictures. Six persons

2 Acer Iconia Tab A510

3 Samsung Galaxy S4 mini

4 https://sites.google.com/site/piotrwendykier/software/jtransforms, last access July $3^{\text {rd }}, 2015$

5 We later repeated some of the tests with a Samsung Galaxy S3 mini which however did not lead to significantly different results.

6 http://www.diakoniewerk.at/, last access July $3^{\text {rd }}, 2015$ 
with different kinds of impairments like spasms, Down's syndrome, spina bifida or different forms of developmental retardation took part in our tests ( 2 male, 4 female, aged 22 to 47). The second user test was held in an EDP workshop for people with disabilities of the same facility but located in Hagenberg. Typical activities there are the digitalization of slides as well as the creation of marketing material like business cards. Here also six persons (2 female, 4 male, aged 21 to 50) with a wide range of impairments, e.g., cerebral palsy, spastic tetraplegia, hydrocephalus, and speech disorders participated. This diverse set of impairments is especially helpful for our project as we do not only aim at assisting one group of people, e.g., with cerebral palsy, but rather adapt existing or recommend new input modalities to each individual user based on their needs. Each test lasted for five to ten minutes; instructions were given by the project team members and are described below.

\subsection{Tasks and Results}

For all tasks, we asked users to apply pressure to the phone, to apply the maximum pressure possible, and to vary pressure if possible. Subsequent illustrations in this section intend to demonstrate how well pressure could be varied. Thereby, the maximum pressure was set to be decently achievable by people without impairments and the minimum pressure was adjusted to equal no interaction at all.

For the vibration test we instructed participants to apply pressure to the smart phone (no specified target area) with their fingers or hands while pushing it against a horizontal surface. For most participants the phone was placed on the desk of their work station (as they could not hold it without assistance and concurrently apply controlled pressure to it). It was up to them to press the phone bimanually or single-handed. The results indicate that in the first test (participants P1-P6) most were able to achieve high levels of pressure whereas in the second test no participant was able to apply the maximum detectable pressure. This might be caused by $i$ ) the relatively high amount of pressure the prototype is configured to regard as maximum and $\mathrm{ii}$ ) the different types of disabilities the participants have, e.g., P12 (Fig. 1 (middle)) could only use one finger whereas P4 (Fig. 1, left) could perform the task bimanually.
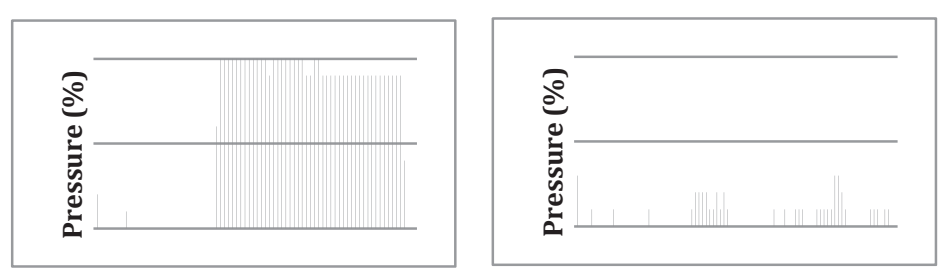

\begin{tabular}{|c|c|c|c|}
\hline Part & Max & Part & Max \\
\hline P1 & 10 & P7 & 2 \\
\hline P2 & 10 & P8 & 5 \\
\hline P3 & 9 & P9 & 8 \\
\hline P4 & 10 & P10 & 4 \\
\hline P5 & 10 & P11 & 5 \\
\hline P6 & 6 & P12 & 3 \\
\hline
\end{tabular}

Fig. 1. Physical pressure applied by participant P4 (left) and P12 (middle), measured with the vibration test. $100 \%$ (y-axis) denotes maximum pressure as measurable by the app over time (x-axis). Right: maximum achieved pressure of all participants.

For the magnetic field test, participants were asked to apply pressure to the mentioned stylus. The pen had to be placed on a circular area on the touch screen. The results show clearly that compared to the vibration method the maximum pressure level was more easily reached by 
the majority of the participants (see an example in Fig. 2). This is most likely due to the fact that the force necessary to press the stylus to its maximum position is lower compared to the force that has to be applied to the device when using the vibration method. However, this approach entails the risk that participants with lower fine-motor skills are only able to apply either maximum or no pressure at all (see P1 in Fig. 2 (right)). Still, keeping a moderate level of pressure for a while was possible for most participants (see an example in Fig. 2 (left)).
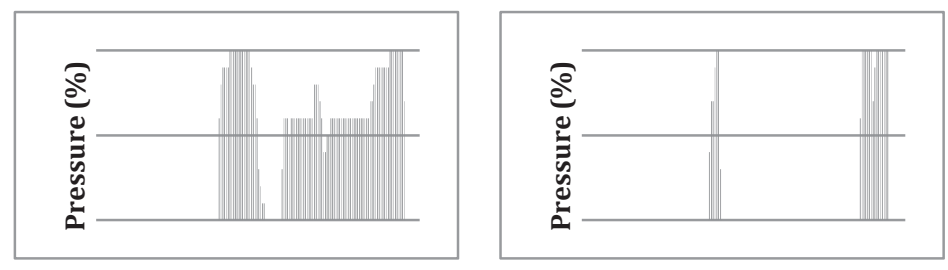

\begin{tabular}{|c|c|c|c|}
\hline Part & Max & Part & Max \\
\hline P1 & 10 & P7 & 10 \\
\hline P2 & 10 & P8 & 10 \\
\hline P3 & 9 & P9 & 10 \\
\hline P4 & 8 & P10 & 10 \\
\hline P5 & 10 & P11 & 8 \\
\hline P6 & 10 & P12 & 10 \\
\hline
\end{tabular}

Fig. 2. Physical pressure applied by participant P5 (left) and P1 (middle) over time (x-axis), using the magnetic field test. Right: maximum achieved pressure of all participants.

For the microphone test, participants were asked to cover the built-in microphone of the smart phone with one finger and apply pressure. Participants with better fine-motor skills were better able to achieve and hold the maximum detectable amount of pressure (see P5 in Fig. 3 (left)), because here it is also necessary to precisely hit the microphone. People with only moderate fine-motor skills were in many cases also not able to apply pressure in a constant manner (mostly because they left the target area), see P10 in Fig. 3 (right). Additionally, many participants needed assistance holding the smart phone while pressing the microphone.
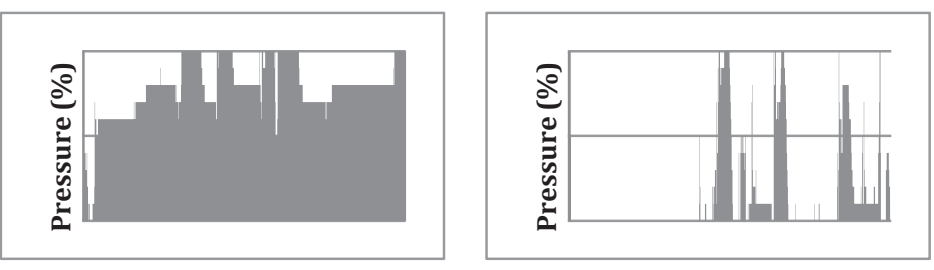

\begin{tabular}{|c|c|c|c|}
\hline Part & Max & Part & Max \\
\hline P1 & 10 & P7 & 10 \\
\hline P2 & 10 & P8 & 10 \\
\hline P3 & 9 & P9 & 10 \\
\hline P4 & 10 & P10 & 10 \\
\hline P5 & 10 & P11 & 10 \\
\hline P6 & 10 & P12 & 10 \\
\hline
\end{tabular}

Fig. 3. Physical pressure applied over time ( $x$-axis) by participant P5 (left) and P10 (middle), using the microphone approach ${ }^{7}$. Right: maximum achieved pressure of all participants.

For the built-in touch test we asked participants to apply pressure to a specified area of the smart phone's touch screen (depicted by a red circle), using one of their fingers. This method partly brought up surprising results. The majority of the participants seemed not to be able to vary the pressure in a controlled manner. The measured pressure seemed to be quite constant for most participants of the second test group. This might be caused by different reasons.

7 Please note that the initial jump (denoting maximum pressure) in both diagrams is caused by a technical shortcoming. 
First, most people in the second group had worse fine motor skills, so many could not vary pressure in a controlled way. Second, people with paralyses were not able to bend their fingers which left the touch area almost unchanged although the pressure actually varied (this was also confirmed by observation protocols). Participants of the first test group (most with better fine-motor skills) were better able to vary and control the amount of pressure but had more difficulty reaching the maximum pressure (not only due to participants' interaction capabilities but also to the technological shortcomings related to this approach).

\section{Conclusions and Future Work}

In this paper we discussed four possibilities of measuring physical pressure with common smart phones and presented the findings of an initial user test with the target group. The main purpose behind this work is to later be able to extend the set of possible interaction modalities for people with motor and/or cognitive impairments and to personalize the overall interaction based on the recorded interaction data. The idea of using physical pressure is rooted in the results of an earlier user observation where we aimed at identifying interactions our target group is capable of performing. We thus compared different technologies that enable us to measure pressure. We did not yet evaluate user interface aspects related to our prototypes because they were not yet intended to be used by the participants autonomously. The findings of our initial study can be summarized as follows: Using the vibration test, most participants were able to perform the task without help because there they could apply pressure to any part of the smart phone (without the need to hit a specified target area). Furthermore, the majority of the participants could relatively well vary pressure. Participants with limited fine motor skills were, however, not able to reach the maximum pressure the device was configured to record. We do not consider this a major problem because this could be adapted to meet the needs of the individual later. We consider this approach useful for our target group and will further investigate its potential for everyday interaction. Using the magnetic field test, we found that participants with comparatively high fine motor skills could handle it remarkably well. For others it was hard to apply different pressure intensities (mostly ending up in maximum pressure or no pressure at all). An additional problem here was that for some participants it was almost impossible to hold the stylus without help. The main shortcoming of this approach lies, however, in the necessity to calibrate the app every time before using it. To overcome the stylus problem we introduced a different input device after the user tests: we built a prototype where the magnet is fixed to a conventional hole puncher - a device that is easier to handle for the target group - which is placed next to the smart phone. The problem regarding calibration remains, we however decided to further work on an automated calibration mechanism that could replace the manual one. We decided not to pursue integration of the microphone test in future prototypes for several reasons. First, it was impossible for several participants to hit the microphone reliably without help (and if, they occasionally left the target area). Second, the frequency to be used varies from device to device and in case the sound can be heard by the user, it is perceived as unpleasant. Third, we found this approach to be highly prone to be disturbed by ambient noise, especially for lower frequency ranges. Regarding the built-in touch test, we also decided not to further 
consider it as it does not reliably work due to its strong dependence on the device. In the worst case, where the developer only receives a binary value (touch or no touch), the desired functionality of measuring pressure intensity would be lost. Current endeavors involve the extension of our app so that it can be used to identify the number of different pressure levels a user can reliably apply. The results will be used to decide what interaction(s) that until now cannot be performed by the user could be replaced by pressure application.

\section{References}

Do, H., \& Silverman, H. F. (2008). A method for locating multiple sources using a frame of a large-aperture microphone array data without tracking. Proceedings of the IEEE Conference on Acoustics, Speech, and Signal Processing (ICASSP). Las Vegas.

Goel, M., Wobbrock, J., \& Patel, S. (2012). GripSense: Using Built-In Sensors to Detect Hand Posture and Pressure on Commodity Mobile Phones. In Proc. of UIST, (2012), 545-554.

Hwang, S., \& Wohn, K. Y. (2012). PseudoButton: Enabling Pressure-Sensitive Interaction by Repurposing Microphone on Mobile Device. CHI12 Extended Abstracts on Human Factors in Computing Systems. Austin, Texas, USA.

Hwang, S., Bianchi, A., \& Wohn, K. Y. (2013). VibPress: Estimating Pressure Input Using Vibration Absorption on Mobile Devices. Proceedings of the 15th International Conference on Human-Computer Interaction with Mobile Devices and Services. Munich, Germany.

Hwang, S., Bianchi, A., Ahn, M., \& Wohn, K.-y. (2013). MagPen: Magnetically Driven Pen Interaction On and Around Conventional Smartphones. Proc. of the 15th Int. Conf. on Human-Computer Interaction with Mobile Devices. Munich, Germany.

Liang, R.-H., Cheng, K.-Y., Su, C.-H., Weng, C.-T., Chen, B.-Y., \& Yang, D.-N. (2012). GaussSense: Attachable Stylus Sensing Using Magnetic Sensor Grid. Procedding of UIST, , (pp. 319-326).

Misra, A., Essl, G., \& Rohs, M. (2008). Microphone as Sensor in Mobile Phone Performance. In Proceedings of the International Conference for New Interfaces Musical Expression (NIME-08). Genova, Italy.

Wilson, G., Stewart, C., \& Brewster, S. A. (2010). Pressure-based menu selection for mobile devices. MobileHCI '10 Proceedings of the 12th international conference on Human computer interaction with mobile devices and services (pp. 181-190). New York, NY, USA: ACM.

\section{Contact Information}

Mirjam Augstein, Daniel Kern, Thomas Neumayr, Werner Kurschl, Josef Altmann, University of Applied Sciences Upper Austria, Softwarepark 11, 4232 Hagenberg \{firstname.lastname\} @fh-hagenberg.at 